\title{
A New Type of Detector for Electron Microscopy
}

\author{
Nguyen-Huu Xuong ${ }^{\mathrm{a}}$, Anna-Clare Milazzo ${ }^{\mathrm{a}}$, Philippe Leblanc ${ }^{\mathrm{a}}$, Fred Duttweiler ${ }^{\mathrm{a}}$, Liang \\ Jin $^{\mathrm{a}}$, James C. Bouwer ${ }^{\mathrm{a}}$, Steve Peltier ${ }^{\mathrm{a}}$, Mark Ellisman ${ }^{\mathrm{a}}$, Stuart Kleinfelder ${ }^{\mathrm{b}}$ \\ ${ }^{a}$ Univ. of California at San Diego, 9500 Gilman Dr., La Jolla, CA 92093, USA \\ ${ }^{\mathrm{b}}$ Univ. of California at Irvine, Irvine, CA 92697, USA
}

\begin{abstract}
A new high resolution recording device for transmission electron microscopy (TEM) is urgently needed. Neither film nor CCD cameras are systems that allow for efficient 3-D high resolution particle reconstruction. We tested an active pixel sensor (APS) array as a replacement device at 200, 300, and $400 \mathrm{keV}$ using a JEOL JEM-2000 FX II and a JEM4000 EX electron microscope. For this experiment, we used an APS prototype with an area of 64 x 64 pixels of $20 \mu \mathrm{m}$ x $20 \mu \mathrm{m}$ pixel pitch. Single electron events were measured by using very low beam intensity. The histogram of the incident electron energy deposited in the sensor shows a Landau distribution at low energies, as well as unexpected events at higher absorbed energies. After careful study, we concluded that backscattering in the silicon substrate and re-entering the sensitive epitaxial layer a second time with much lower speed caused the unexpected events. Exhaustive simulation experiments confirmed the existence of these back-scattered electrons. For the APS to be usable, the backscattered electron events must be eliminated, perhaps by thinning the substrate to less than $30 \mu \mathrm{m}$. By using experimental data taken with an APS chip with a standard silicon substrate $(300 \mu \mathrm{m})$ and adjusting the results to take into account the
\end{abstract}


effect of a thinned silicon substrate $(30 \mu \mathrm{m})$, we found an estimate of the signal-to-noise ratio for a back-thinned detector in the energy range of $200-400 \mathrm{keV}$ was about 10:1 and an estimate for the spatial resolution was about $10 \mu \mathrm{m}$. We will also report on our efforts to reduce the pixel size to $5 \mu \mathrm{m} \times 5 \mu \mathrm{m}$. 